\title{
Bottom Topography as a Control Variable in an Ocean Model
}

\author{
Martin Losch AND CARL Wunsch \\ Department of Earth, Atmospheric, and Planetary Sciences, Massachusetts Institute of Technology, Cambridge, Massachusetts
}

(Manuscript received 13 August 2002, in final form 10 March 2003)

\begin{abstract}
The possibility of using topography in a state estimation context as a control parameter is explored in a linear barotropic shallow water model. Along with its adjoint, the model is used to systematically assess the influence of the depth field on the modeled circulation in a steady state. Sensitivity of the flow field to the topography is greater in a partially blocked zonal channel than in a subtropical gyre. Hypothetical surface elevations are used to represent the types of data actually available. In neither case can all the details of the topography be recovered, showing that the relationship between topography and elevation does not have a unique inverse, and that many details of the topography are irrelevant to the particular physics under consideration.
\end{abstract}

\section{Introduction}

Numerical ocean models produce results dependent upon many parameters and parameterizations, including diffusion coefficients, wind forcing, lateral boundary conditions, initial conditions, and many others. Much activity is directed at understanding the sensitivity of the results to a subset of these parameters by comparisons to observations. An organized form of testing the sensitivity goes by the name of data assimilation or state estimation. When done rigorously (e.g., Stammer et al. 2002; Wunsch 1996), certain fields and parameters are identified as "control parameters." The control parameters are independent variables that are systematically modified within stipulated limits to bring the model within an estimated error of the observations.

The introduction of a field as a control variable implies the belief that the model result (trajectory through its phase space) is indeed sensitive to that field in some way that is important to reproducing the observations. "Controllability" is the mathematical concept underlying the belief that a variable is likely to be important (see, e.g., Wunsch 1996, p. 383). For a general circulation model (GCM), no full study of controllability or the related concept of "observability" has ever been carried out, as it involves potentially very difficult questions of model differentiability and the effective rank of very large matrices. Instead, plausible assumptions have been made about the dominant control variables, and these have become the focus of most state estimation efforts. Thus initial conditions and surface

Corresponding author address: Martin Losch, Alfred-Wegener-Institut für Polar- und Meeresforschung, Postfach 120161, 27515 Bremerhaven, Germany.

E-mail: mlosch@awi-bremerhaven.de boundary conditions have been the primary control fields for GCMs (e.g., Stammer et al. 2002).

In this paper, we begin the exploration of the importance, and practicality, of using bottom topography as controls in models of the ocean circulation. The results are envisioned as only the first step toward exploration of the problem in more complex models, and also toward employing, for example, lateral boundary conditions (free-slip, no-slip, hyper-slip, etc.) as fields to be determined rather than assumed a priori.

Bottom topography plays a major role in determining the flow field in the ocean. It is, however, inaccurately known in many regions, and even where accurately known, the best way to represent it in ocean models is obscure. For large regions of the Southern Ocean or the Arctic Sea, one relies on charts of bottom topography that were derived from very few in situ measurements. Different map products sometimes describe very different bottom topographies. For example, the Foundation Seamounts in the South Pacific were unknown prior to space-borne altimetry (Smith and Sandwell 1997) and are consequently not represented in the frequently used dataset ETOPO5 (NOAA 1988). The bathymetry of Smith and Sandwell (1997) is a major step forward in constructing a global topography database for the ocean, but the assessment of the absolute accuracy of topography still remains difficult (W. H. F. Smith 2001, personal communication).

Numerical GCMs have limited resolution and the representation of ocean basin geometry can be crude. Even an accurate discretization of a perfectly known bathymetry may not lead to the best possible computed flow field. As a common assumption, one concentrates on the large-scale components of the flow, which presumably "feel" only the corresponding large-scale com- 
ponents of the bottom topography, a representation of which can be obtained by smoothing the real geometry (Il' in et al. 1974). As with subgrid-scale mixing processes, the influence of small-scale features has to be parameterized. But little or nothing appears to be known about how to do it apart from the use of some forms of simplified "wave drag" (e.g., McFarlane 1987). Whether the best fit of a coarse resolution model to real bathymetry is a straightforward average or another approximation to the real topography is one of the issues that must be addressed.

Similar ideas about the influence of bottom topography have given rise to studies of its use as control parameters in shallow water models of shelf seas (Das and Lardner 1991, 1992; Heemink et al. 2002; Lardner et al. 1993; ten Brummelhuis et al. 1993) using a variety of optimization methods in various approximations. The focus has tended to be on tidal modeling, whose gravity wave dynamics are quite distinct from the nearly geostrophic limit of most open ocean general circulation models.

The use of control methods to systematically adjust dynamical models is now a familiar, if not yet completely common, endeavor. That such methods "work," in the sense that solutions can be found if the data and model are consistent, is not in doubt. The questions being addressed here are 1) whether the dynamics peculiar to the large-scale ocean circulation and the particular way in which topography enters the equations of motion causes any unexpected difficulties, and 2) to the extent that sensitivities and uncertainties about the topography remain, to try and understand the physical causes. All so-called inverse methods ultimately solve an optimization problem; thus in question 1, the concern is most often about the ability to numerically reduce a potentially very complex objective or cost function.

Estimating topography from data involves a nonlinear optimization problem because the dynamic variables depend nonlinearly on depth. In this light, it is important to understand whether, given the available data, there is a unique optimal representation of topography, or whether there are many different representations equivalently consistent with the observations. We address this question systematically in a model of the ocean circulation by invoking inverse (state estimation) methods.

Ultimately, we are interested in the topographic representation in a full model. As a starting point, the study is here confined to a linear shallow water model in steady state. In addition to the information contained in the model dynamics and in the hypothetical data, the realistic assumption is made that useful a priori estimates of the topography are available as well.

The steady-state flow, in two distinct dynamical regimes, is explored: (a) a zonal channel, whose dimensions are based on previous models of the Antarctic Circumpolar Current (ACC); and (b) in a midlatitude gyre flow. Different sensitivities to bottom topography can be anticipated in the two configurations.
The only "observations" used here are sea surface height (altimeter) data. These pseudo data are the natural choice because they are the only available observable physical property reflecting the three-dimensional, large-scale fluid flow (Wunsch and Stammer 1998). The uncertainties associated with existing absolute sea surface height data are still large because of geoid errors, but new gravity missions will reduce them by an order of magnitude (Ganachaud et al. 1997; LeGrand 2001; Schröter et al. 2002).

The paper is organized as follows. Section 2 describes the shallow water model and both analytic and control theory approaches toward exploring the sensitivity of the flow to bottom topography. In section 3, it is demonstrated how the sensitivity of an objective function to infinitesimal perturbations can be assessed. In twin experiments in sections 4,5 , and 6 , the shallow water flow is constrained by sea surface height data in various configurations. A discussion and conclusions are given in section 7 .

\section{Linear shallow water model}

\section{a. Model domain and control run}

The equations used are for linearized shallow water flow on the $\beta$ plane without horizontal friction and with a linear parameterization of bottom stress. Their primitive form is represented in finite differences on a $\mathrm{C}$ grid. Starting from initial conditions for velocity and sea surface height, the equations are stepped forward in time by a scheme that treats the bottom stress term implicitly and the Coriolis term explicitly.

As a first step toward understanding the role of bottom topography, consider the flow in a periodic zonal channel with solid boundaries to the north and the south on a $\beta$ plane with idealized topography. The scale is based on that of the ACC, with length $X=4000 \mathrm{~km}$, width $Y=1600 \mathrm{~km}$, and maximum depth $H_{0}=4 \mathrm{~km}$. The horizontal grid cell length is $\Delta x=\Delta y=200 \mathrm{~km}$ resulting in $20 \times 8=160$ grid cells. Here $f_{0} \approx-1.2 \times$ $10^{-4} \mathrm{~s}^{-1}$ and $\beta \approx 1.3 \times 10^{-11} \mathrm{~s}^{-1} \mathrm{~m}^{-1}$ are chosen such that the channel is centered at approximately $55^{\circ} \mathrm{S}$. The flow is forced with steady eastward winds exerting a stress, $\tau^{x}=\tau_{0} \sin \pi y / Y$, with $\tau_{0}=10^{-4} \mathrm{~m}^{2} \mathrm{~s}^{-2}$. The bottom friction parameter $r=4 \times 10^{-3} \mathrm{~m} \mathrm{~s}^{-1}$ is chosen so that the spindown time is $H_{0} / r \approx 11.6$ days.

A central meridional Gaussian sill extends across the channel with a crest $400 \mathrm{~m}$ above the maximum depth of $4000 \mathrm{~m}$. Its half-width of $200 \mathrm{~km}$ corresponds to an average slope of $1 \mathrm{~m}$ in $1000 \mathrm{~m}$. The depth $h$ is shown in Fig. 1.

The model is spun up to a steady state with a time step of $100 \mathrm{~s}$ for 60000 time steps $(\approx 70$ days). Sea surface height and velocity of this steady state, together with the $f / h$ contours are shown in Fig. 2. For illustrative purposes, the steady-state equations of motion can be written in terms of a transport streamfunction $\psi$, 


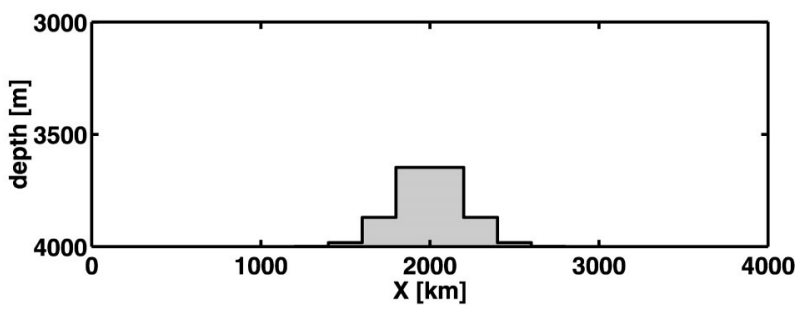

FIG. 1. A section along the zonal channel with the Gaussian sill.

$$
\begin{aligned}
J\left(\psi, \frac{f}{h}\right)= & \mathbf{k} \cdot \operatorname{curl}\left(\frac{\boldsymbol{\tau}}{h}\right) \\
& -r\left\{\frac{\partial}{\partial x}\left(\frac{1}{h^{2}} \frac{\partial \psi}{\partial x}\right)+\frac{\partial}{\partial y}\left(\frac{1}{h^{2}} \frac{\partial \psi}{\partial y}\right)\right\},
\end{aligned}
$$

where the term on the left-hand side is the Jacobian and $\boldsymbol{\tau}=\left(\tau^{x}, 0\right)$. In the absence of bottom stress and forcing, $J(\psi, f / h)=0$, implying that the streamlines coincide with the geostrophic contours $f / h$. Dissipation and surface stress force the flow across geostrophic contours (Pedlosky 1987). To within the quasigeostrophic approximation, the elevation $\eta$ and the scaled streamfunction $(f / g) \psi$ are the same.

Here, the sill deflects the $f / h$ contours equatorward where they are blocked at the northern boundary of the channel. The number of blocked contours increases with the height of the sill, and hence controls the transport through the channel.

Note that although the $f / h$ contours are symmetric about the center of the channel, the stress terms in the momentum equations break the symmetry, so that the maximum northward deflection of the flow is shifted downstream from the corresponding position of the geostrophic contours. Both the strongest and the weakest flow near the sill occur slightly downstream of the sill crest.

\section{b. Parameter estimation: Analytical approach}

Assuming only zonal wind stress, Eq. (1) can be recast as a partial differential equation for the inverse depth $\alpha=h^{-1}$ :

$$
\begin{aligned}
& \left(r \nabla^{2} \psi\right) \alpha+2 r \nabla \psi \cdot \nabla \alpha+f J(\psi, \ln \alpha)-\tau^{x} \frac{\partial}{\partial y} \ln \alpha \\
& =\frac{\partial \tau^{x}}{\partial y}-\beta \frac{\partial \psi}{\partial x} .
\end{aligned}
$$

The equation is nonlinear in $\alpha$ owing to the presence of the logarithm. If the streamfunction $\psi$, or surface elevation, and the boundary conditions for $\alpha$ are known, Eq. (2) has a solution for given $\psi$, which could be found using numerical methods.

Some additional insight into the problem can be obtained by linearization. To do so, denote dimensional
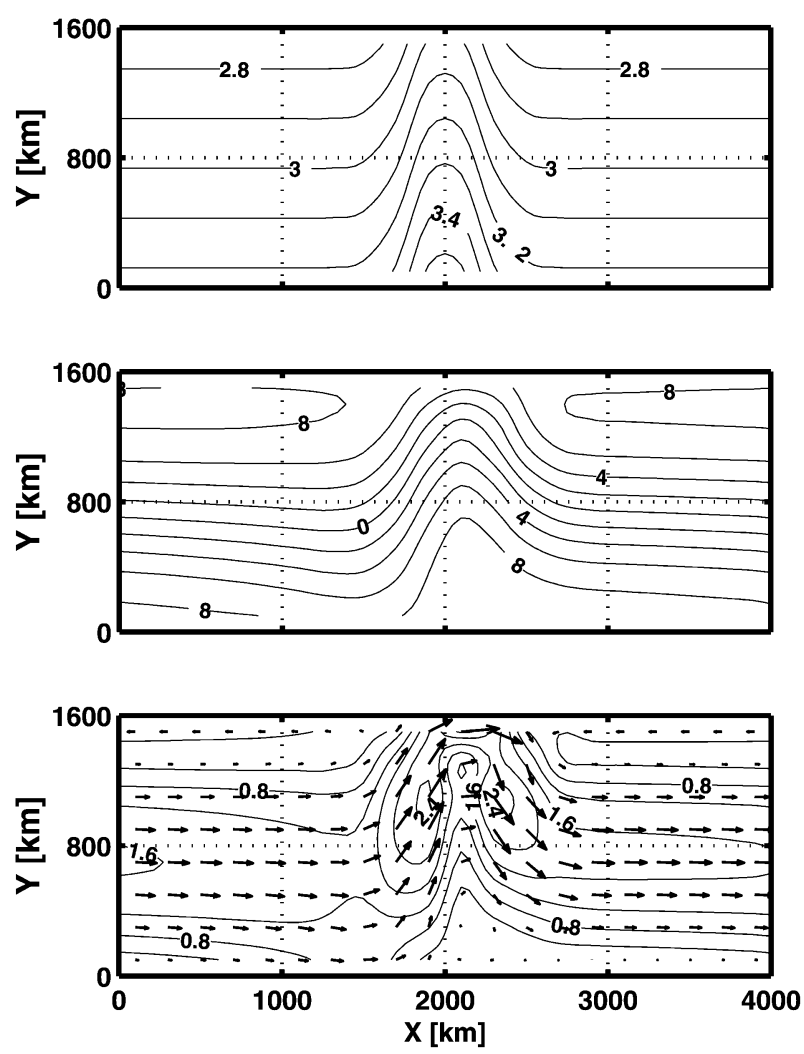

FIG. 2. (top) The $f / h$ contours of the channel (contour interval is $\left.0.1 \times 10^{-8} \mathrm{~s}^{-1} \mathrm{~m}^{-1}\right)$. (Quasi-) steady-state flow field after spinup of $\approx 70$ days: (middle) sea surface height (contour interval is $2 \mathrm{~cm}$ ) and (bottom) velocity (contours display the current speed, arrows its direction; contour interval is $0.2 \mathrm{~cm} \mathrm{~s}^{-1}$ ).

variables temporarily with asterisks, and define nondimensional ones,

$$
\begin{aligned}
x_{*}=X x, & y_{*}=X y, \quad \delta=A / H_{0} \\
f_{*}=f_{0} f=f_{0}\left[1+\beta\left(y-\frac{a}{2}\right)\right], & \text { with } \\
\beta=\frac{X}{f_{0}} \beta_{*}, & h_{*}=H_{0}(1+\delta \xi), \\
\tau_{*}=\tau_{0} \boldsymbol{\tau}, & \epsilon=\frac{r}{f_{0} H_{0}},
\end{aligned}
$$

where $\xi$ is an $O(1)$ nondimensional topography, $A$ a scale for the relief height, so that $\delta \ll O(1)$, and $a=Y / X$ is the domain aspect ratio. With the velocity scale

$$
U=\frac{r_{0}}{f_{0} H_{0}}, \quad \psi_{*}=\frac{U H_{0} Y}{a} \psi,
$$

and, for small $\delta$,

$$
\begin{aligned}
(1+\delta \xi)^{-1} & =1-\delta \xi+O\left(\delta^{2}\right), \\
\psi & =\bar{\psi}+\delta \psi^{\prime} .
\end{aligned}
$$

Neglecting terms of $O\left(\delta^{2}\right)$, Eq. (1) becomes 


$$
\begin{gathered}
\left(2 \epsilon \frac{\partial \bar{\psi}}{\partial x}-f \frac{\partial \bar{\psi}}{\partial y}+\tau^{y}\right) \frac{\partial \xi}{\partial x}+\left(2 \epsilon \frac{\partial \bar{\psi}}{\partial y}+f \frac{\partial \bar{\psi}}{\partial x}-\tau^{x}\right) \frac{\partial \xi}{\partial y} \\
-\left(\epsilon \nabla^{2} \bar{\psi}\right) \xi=-\left(\epsilon \nabla^{2} \psi^{\prime}+\beta \frac{\partial \psi^{\prime}}{\partial x}\right)
\end{gathered}
$$

after choosing an unperturbed state $\bar{\psi}$ of flow over a flat bottom, for which

$$
\epsilon \nabla^{2} \bar{\psi}+\beta \frac{\partial \bar{\psi}}{\partial x}-\mathbf{k} \cdot \operatorname{curl} \boldsymbol{\tau}=0 .
$$

For a wind field $\boldsymbol{\tau}=(\sin \pi y / a, 0), \bar{\psi} \propto[1-\cos (\pi y / a)]$, and Eq. (7) simplifies to

$$
\begin{aligned}
& \sin \frac{\pi}{a} y\left(\frac{\partial \xi}{\partial y}-\frac{f}{\epsilon} \frac{\partial \xi}{\partial x}\right)+\left(\frac{\pi}{a} \cos \frac{\pi}{a} y\right) \xi \\
& =\left(\epsilon \nabla^{2} \psi^{\prime}+\beta \frac{\partial \psi^{\prime}}{\partial x}\right) .
\end{aligned}
$$

Equations (7) and (9) are linear first-order partial differential equations in the perturbation $\xi$ to the flat bottom case, which can be solved by the method of characteristics or numerically, given a set of starting values of $\xi$.

Rather than pursuing this analytical approach, we seek instead a more flexible method capable of dealing with the eventually much more complex wind fields, finite-amplitude topography, and ultimately baroclinic physics of a GCM, as well as the errors in measurements of $\psi$. Equations (2), (7), or (9) do, however, show that the control problem is equivalent to the determination of the depth field from a set of observables $\psi$. Because the streamfunction $\psi$ cannot be observed directly in a realistic configuration, other types of data must be used, for example, observations of sea surface height, which are the same as $(f / g) \psi$ within the geostrophic approximation. It is apparent that all of the issues of continuity, differentiability, etc., that arise for the solutions of partial differential equations will have their counterparts in the control problem.

\section{c. Parameter estimation: Objective function and adjoint model}

As discussed, for example, in Wunsch (1996), there is a close connection between so-called Gauss-Markov or minimum variance estimation, and the solution of an equivalent least squares problem. In this paper, we will use the language and formalism of least squares, recognizing, however, that the interpretation best placed on the result is that of a solution to a statistical estimation problem.

Let $y_{i}=\mathcal{H}_{i}(\boldsymbol{\psi})+n_{i}$ represent the pseudo observations, having noise element $n_{i}$, supposed to have zero mean; let $\mathbf{y}=\mathscr{H}(\boldsymbol{\psi} \boldsymbol{s})+\mathbf{n}$ be the vector of observations, and $\mathbf{n}$ the vector of noise. Here $\mathcal{H}$ is a general operator that maps the state vector $\boldsymbol{\psi}$ to the observations. The least squares approach demands the depth field for which a quadratic objective function of the type (e.g., Wunsch 1996)

$$
\mathcal{J}=\frac{1}{2}[\mathbf{y}-\mathcal{H}(\boldsymbol{\psi})]^{\mathrm{T}} \mathbf{C}_{n}^{-1}[\mathbf{y}-\mathcal{H}(\boldsymbol{\psi})]+\text { other terms }
$$

has an acceptable global minimum. Matrix $\mathbf{C}_{n}$ describes the prior error covariance estimate. Because the model state, $\psi$, is a nonlinear function of the field $h$ (all asterisks are removed, and all variables are dimensional from now on), the least squares problem is also nonlinear. Starting from an initial estimate, a quasi-Newton algorithm (Gilbert and Lemaréchal 1989) at each step evaluates the gradient of the objective function (10) with respect to depth and calculates a new state based on the gradient information until a convergence criterion is met. The gradient field is found through the so-called adjoint model (e.g., Wunsch 1996), which is generated semi-automatically by the Transformation of Algorithms in FORTRAN (TAF) compiler (Giering and Kaminski 1998; Marotzke et al. 1999) applied to the FORTRAN source code of the forward model.

Assuming that one has found the global minimum, the Hessian matrix of second derivatives of the objective function $g$ at the minimum can be computed with code that has also been generated by TAF. Let $\mathcal{M}(h)=$ $\mathcal{H}[\psi(h)]$ be the nonlinear model operator that maps the depth $h$ to the data, then the linearized Hessian $\mathbf{H}$ (not to be confused with the observation operator $\mathcal{H}$ ) is

$$
\mathbf{H}=\left(\frac{\partial \mathcal{M}}{\partial \mathbf{h}}\right)^{\mathrm{T}} \mathbf{C}_{n}^{-1}\left(\frac{\partial \mathcal{M}}{\partial \mathbf{h}}\right)
$$

where the Jacobian or adjoint model operator $(\partial \mathcal{M} / \partial \mathbf{h})_{i j}^{\mathrm{T}}=\left(\partial \mathcal{M}_{i} / \partial h_{j}\right)^{\mathrm{T}}$ is evaluated at the minimum.

In the neighborhood of the solution, the inverse Hessian is proportional to the covariance matrix of the solution. Hence the eigenvalues of the Hessian can be used to determine which components of the control parameter are well or poorly determined by the data (Thacker 1989), with small eigenvalues corresponding to poorly determined components and vice versa. Zero eigenvalues correspond to completely indeterminate elements that lie in the problem null space. (Note that the concept of a null space is only directly applicable in the linearized problem. At finite amplitude, the indeterminate components are constructed here as the difference between the known correct solution, and the one found by nonlinear optimization.)

\section{Sensitivity of zonal volume transport to depth}

The adjoint model permits calculation of the gradient of any parameter, or combination of parameters, with respect to the controls. Thus the sensitivity of the solution element to perturbations in control parameters is readily found. Consider, briefly, an alternative scalar 


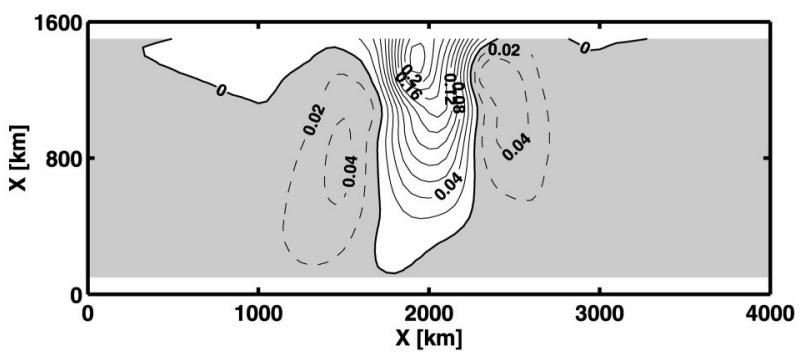

FIG. 3. Gradient of zonal transport with respect to depth $\left(\mathrm{Sv} \mathrm{m}{ }^{-1}\right)$. Areas of negative values are shaded. Contour interval is $0.02 \mathrm{~Sv} \mathrm{~m}^{-1}$.

observable that can be used to characterize the flow. The zonal volume transport,

$$
T_{V}=\int_{0}^{Y} h\left(x_{0}, y\right) u\left(x_{0}, y\right) d y,
$$

depends explicitly and implicitly on the topography in the channel, but is independent of the zonal position $x_{0}$. The gradient of the zonal transport with respect to depth $\partial T_{V} / \partial h$ is the spatial distribution of this sensitivity (Fig. $3)$. Sensitivity is largest over the northern end of the sill where most of the flow traverses the obstacle. Here, decreasing the height of the sill at one grid point by 5 $\mathrm{m}$ would increase the flow by $1 \mathrm{~Sv}\left(=10^{6} \mathrm{~m}^{3} \mathrm{~s}^{-1}\right)$. At the southern end, changes in sill height hardly affect the flow. The gradient of $T_{V}$ with respect to depth is slightly negative in the deep-sea regions away from the sill. Making these regions shallower shifts the $f / h$ contours north, thus moving some of the blocked contours across the northern boundary and out of the model domain. They are replaced by unblocked contours near the southern boundary. More unblocked contours lead to higher transports.

Thus, the gradient systematically assesses the sensitivity of the flow to topography in a quantitative way. Furthermore, this sensitivity can be conveniently connected to the dynamics of the flow. In the following sections we explore the ability to estimate depth from the combined sea surface height data and the model.

\section{Error-free-data experiments}

In this section the method of "identical twins" is used to explore how topography as an independent variable is constrained by sea surface height data. After the model is spun up to a near-steady state, it is integrated for another 40000 time steps ( $\approx 46$ days) to produce pseudo data. In a subsequent run with identical boundary and initial conditions, the model necessarily fits these values perfectly, and the objective function has its minimum value (zero). In bypassing the minimization, we avoid convergence problems at this point. The system is at a global minimum, and we can safely employ the linearization of the Hessian matrix for error calculations.

With a perfect result, the only consistent error esti-

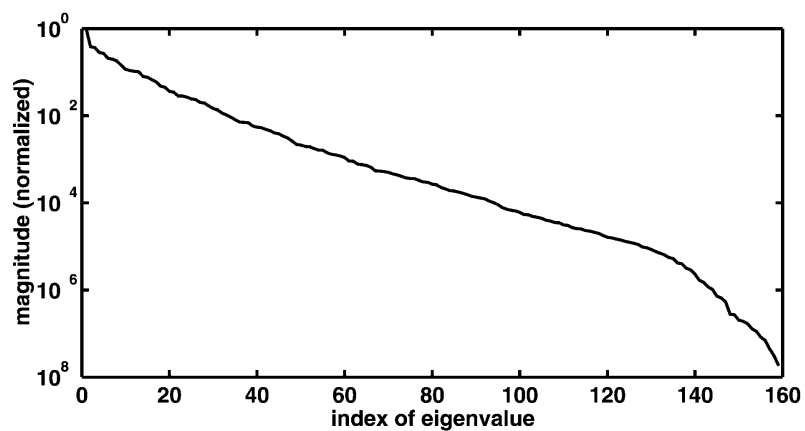

FIG. 4. Eigenvalues of the Hessian of the objective function $\mathcal{I}_{1}$ [Eq. (12)] normalized by the maximum eigenvalue. The smallest eigenvalue, which is numerically zero $\left[O\left(10^{-15}\right)\right]$, is not shown. The corresponding null space vector consists mostly of grid-scale noise.

mate would have zero variance. We can nonetheless explore the impact of data errors on the accuracy of depth estimates, in the limit of small perturbations about the perfect solution. The case where errors are added to the pseudo data will be taken up later (section 5).

We use two different objective functions. In the first, we assume that we do not know anything about the depth, and in the second we use an a priori depth estimate that is accurate to within $200 \mathrm{~m}$. We anticipate that with the first objective function not all depth values can be determined from data (see also Das and Lardner 1992). As an intuitive explanation, consider that in regions of no flow the height of the topography has no effect. A topographic estimate from data in such a quiescent region would necessarily be nonunique.

With the second objective function, a bias toward the a priori depth estimate is introduced into the system.

\section{a. Sea surface height data only}

The first objective function is

$$
\mathcal{J}_{1}=\frac{1}{2}[\mathbf{y}-\mathcal{M}(\mathbf{h})]^{\mathrm{T}} \mathbf{C}_{\eta}^{-1}[\mathbf{y}-\mathcal{M}(\mathbf{h})],
$$

where $y_{i}$ are the sea surface height data, $\mathcal{M}_{i}(\mathbf{h})$ their model counterparts, and $\mathbf{C}_{\eta}=\sigma_{\eta}^{2} \mathbf{I}$ is a diagonal covariance matrix with the constant prior error estimate $\sigma_{\eta}$ for the data. Here $\sigma_{\eta}$ is chosen to be $10 \mathrm{~cm}$. Only relative weights affect the solution of the optimization problem, so that the size of $\sigma_{\eta}$ has, for the moment, only the role of a uniform scaling factor. Later, however, additional, differently weighted terms will be introduced.

An eigenvalue spectrum of the numerical approximation of the Hessian matrix is shown in Fig. 4. The displayed range of the spectrum spans eight orders of magnitude; the smallest eigenvalue, which is numerically zero $\left[O\left(10^{-15}\right)\right]$, is not shown. The null space vector corresponding to the zero eigenvalue shows a nearly pure grid-scale $(2 \Delta x)$ structure.

Eigenvectors corresponding to the very small, but 
nonzero eigenvalues, are also dominated by grid-scale noise. But they also have a superimposed large-scale structure that we believe arises from technical aspects of the numerical scheme, and so we do not attach any physical significance to them. On a $\mathrm{C}$ grid, the kinematically relevant depth at the velocity points has to be found by interpolation between the values at the grid cell centers where the control parameters are located. This interpolation is clearly responsible for the gridscale noise. One could avoid this issue by choosing the depth at the velocity points as control parameters. But this doubles the number of the control parameters. Also, as will be clear later on, introducing prior information for topography eliminates the grid-scale-noise problem.

In an attempt to add information to the system that otherwise lies in the null space, one can augment the data vector $\mathbf{y}$ in Eq. (12) with direct observations of the velocity. But experiments (not shown) reveal that the structure of the null space does not change when one includes in the objective function even a full row of velocity measurements that spans the channel-presumably because the grid noise in the model has a very local structure.

Because of the zero eigenvalue, the condition number, which is the ratio of the largest and the smallest eigenvalue, is infinite and the Hessian matrix is singular, even if one were willing to regard the other very small eigenvalues as being mathematically nonzero. In the presence of any noise at all, some of the small eigenvalues are effectively zero, and one therefore infers immediately that there will, at least in a formal sense, be an infinite number of acceptable solutions to the problem of determining $h$. One needs ultimately, to understand the family of acceptable solutions. In section 5 the shape of the poorly determined components of bottom topography will be discussed.

\section{b. Sea surface height data and prior depth estimate}

The objective function (12) is augmented by a term that penalizes deviations from a prior depth estimate, $h_{0}$ as,

$$
\begin{aligned}
J_{2}= & \frac{1}{2}[\mathbf{y}-\mathcal{M}(\mathbf{h})]^{\mathrm{T}} \mathbf{C}_{\eta}^{-1}[\mathbf{y}-\mathcal{M}(\mathbf{h})] \\
& +\frac{1}{2}\left(\mathbf{h}_{0}-\mathbf{h}\right)^{\mathrm{T}} \mathbf{C}_{h}^{-1}\left(\mathbf{h}^{0}-\mathbf{h}\right),
\end{aligned}
$$

where $\mathbf{C}_{h}=\sigma_{h}^{2}$ I is a, for now, diagonal covariance matrix with the constant prior error estimate $\sigma_{h}$ for depth. With the addition of this term, which renders the problem one of "tapered least squares," the null space, either formal or effective, is suppressed. For simplicity, the prior error estimate for sea surface height is again constant in space and time with standard deviation $\sigma_{\eta}=10 \mathrm{~cm}$. This value is in rough accord with the average combined error of satellite altimetry and an underlying geoid model (Wunsch and Stammer 1998). A large depth error, $\sigma_{h}$

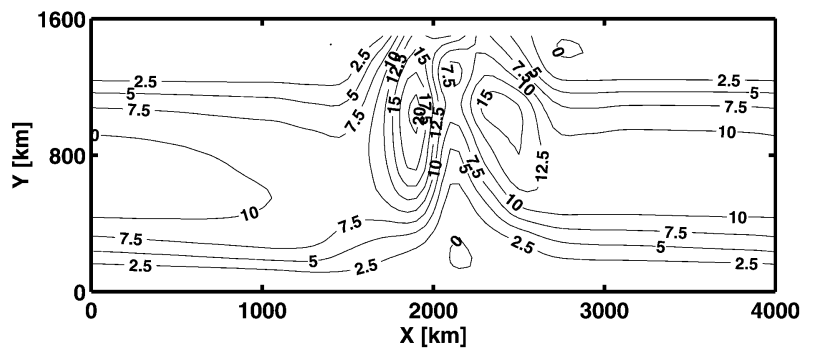

FIG. 5. Estimated error reduction for depth (in $\%$, relative to the prior error estimate $\sigma_{h}=200 \mathrm{~m}$ ); error-free data; deviations from the (true) initial guess are penalized.

$=200 \mathrm{~m}$, is chosen to prevent the prior estimate of the depth from dominating the new estimate. Also, the vertical resolution of a GCM near the bottom can be very poor, so that $\sigma_{h}$ can be interpreted also as the accuracy by which depth is represented on a numerical grid.

The condition number of the Hessian of $g_{2}$ is $\approx 24$, and the problem is well conditioned. None of the eigenvectors contain any grid-scale noise. The posterior relative error reduction estimate $\left(=1-\sqrt{\operatorname{diag}\left(\mathbf{H}^{-1}\right)} / \sigma_{h}\right)$ in percent $(\%)$ is shown in Fig. 5. The impact of sea surface height data on the topography estimate is greatest where the current speeds are highest. Above the flanks of the sill, the topography error estimate is reduced by up to $20 \%$ compared to the initial error estimate of $200 \mathrm{~m}$, while above the southern part of the sill and along the northern and southern boundaries away from the sill there is hardly any error reduction at all.

\section{Recovering the "true depth"}

The experiments of section 4 suggest that measurements of the dynamical properties of the flow are not sufficient to remove the problem null space. Hence, one cannot expect to estimate a unique bottom topography with a shallow water model, unless there is additional information available about those components of the topography lying in the effective null space of the problem. But we are primarily interested in an optimal representation of topography in an ocean model. In this context the null space components of the topography will not affect the flow and need not be determined from data.

In the following experiments, the optimization is started from an incorrect prior depth estimate $h_{0}$ for which the first estimate of the sill height has been incorrectly set to $360 \mathrm{~m}$, that is, with a $10 \%$ initial error. Deviations from this prior estimate are penalized as described by objective function $g_{2}$ [Eq. (13)]. Doing so introduces a bias toward the incorrect prior depth estimate $h_{0}$. By choosing small weights for the penalty term, that is, assuming a large error for the prior depth estimate, one can alleviate the effects of the bias, but cannot remove them entirely. (The prior error estimates 

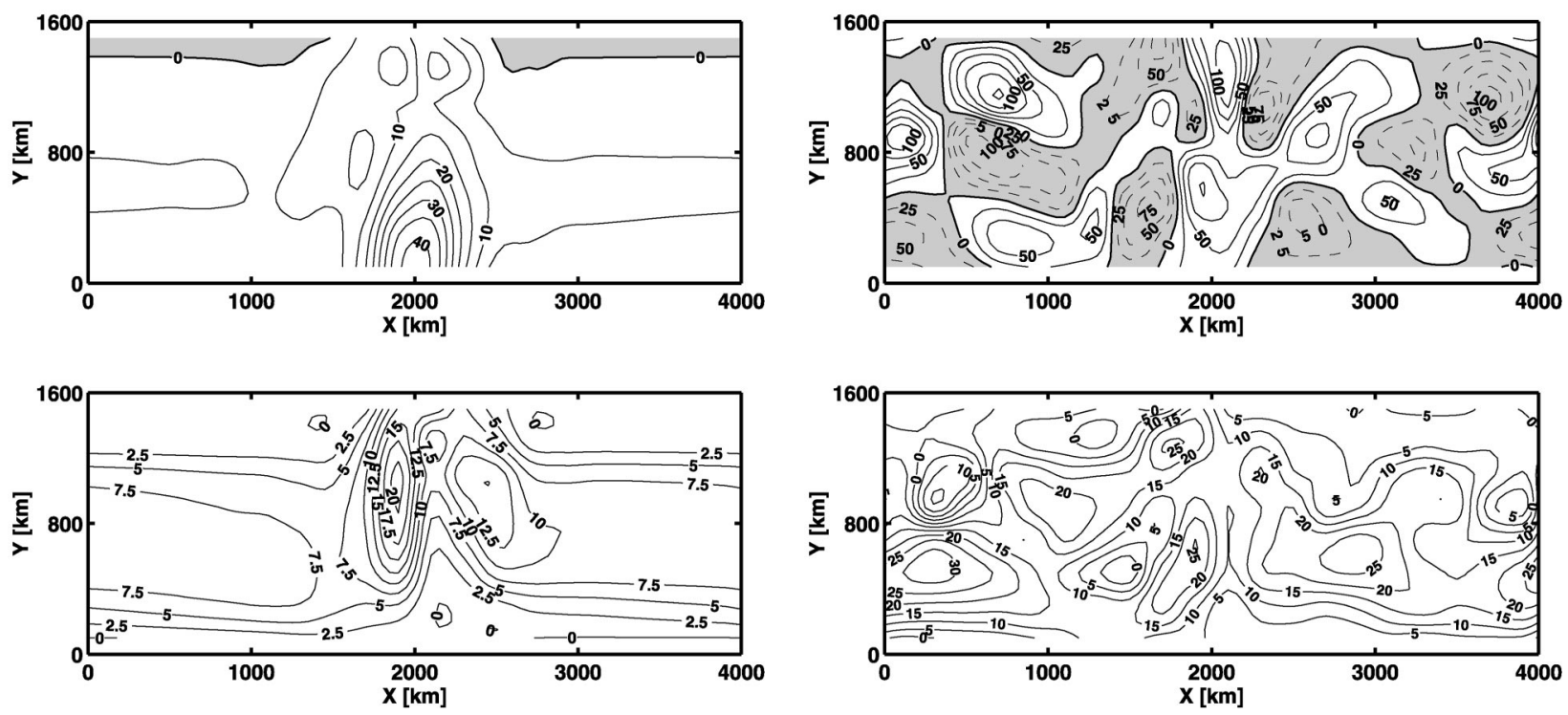

FIG. 6. (top) Difference between true depth and estimated depth (in $\mathrm{m}$ ) and (bottom) estimated error reduction (in \%, relative to the initial error estimate of $200 \mathrm{~m}$ ); perfect sea surface height data; deviations from the (wrong) initial estimate are penalized.

chosen here are the same as in section $4 \mathrm{~b}, \sigma_{\eta}=10 \mathrm{~cm}$, and $\sigma_{h}=200 \mathrm{~m}$.)

\section{a. Perfect sea surface height data and a prior depth estimate}

Figure 6 shows the difference between the true depth and the estimated depth after minimizing the objective function $g_{2}$ with sea surface height data that has been generated with the correct topography. The difference between the estimated depth and the true depth is small over the northern part of the sill where the current velocities are high (cf. Figs. 2 and 6). Over the southern end of the sill, the sea surface height data have little effect, and the differences between the optimal estimate and the true depth are as large as $40 \mathrm{~m}$. The difference between the estimate and the true depth is always smaller than the posterior error estimate, and so the solution is statistically consistent.

\section{b. Noisy sea surface height data and a prior depth estimate}

With objective function $g_{2}$ [Eq. (13)] the problem is formally well determined, but the information provided by the sea surface height data is not sufficient to reconstruct the true depth completely: in the previous section, the estimated depth differs from the true one, in some places by as much as the initial difference.

The problem is now extended to explore the sensitivity of the optimal solution to random Gaussian noise in the data having a standard deviation of $10 \mathrm{~cm}$. With these noisy data, the experiment of the previous section with objective function $g_{2}$ is repeated.

FIG. 7. (top) Difference between true depth and estimated depth (in $\mathrm{m}$ ) and (bottom) estimated error reduction (in \%, relative to the initial error estimate of $200 \mathrm{~m}$ ); noisy sea surface height data; deviations from the (wrong) initial guess are penalized.

When the forward model is run with an incorrect topographic estimate as in section $5 \mathrm{a}$, it produces an rms deviation from the correct sea surface height of only $0.5 \mathrm{~cm}$. After optimization, using the noisy sea surface height data, this rms difference is hardly changed, and the difference between the true depth and estimated depth remains very large (Fig. 7) with an rms difference of $45 \mathrm{~m}$ and a maximum difference of $124 \mathrm{~m}$. Thus major differences in bottom topography generate only very slight differences in surface elevation. After optimization, all differences in surface elevation and bottom topography are smaller than the formal error estimates and the solution is statistically consistent. The error estimate still shows the spatial patterns of Figs. 5 and 6 with smallest errors (largest error reductions) where the velocities are strong, but as a consequence of the random nature of the noise in the data, the depth error reduction can locally be much greater than with perfect data (Fig. 6).

In spite of the noisy topography estimate, the estimate of the sea surface height by the inverse model is improved over the noisy data: the rms difference between the temporal means of the perfect and the noisy data is $1.2 \mathrm{~cm}$; for the perfect data and the model estimate, this rms difference is only $0.5 \mathrm{~cm}$. Therefore, adjusting the topography improves the model-data fit. But the new sea surface height mean (Fig. 8) implies the difference to the true topography shown in Fig. 7. Reducing the noise level in the data leads to a less distorted sea surface height estimate, which in turn reduces the noise in the topography estimate (not shown). 


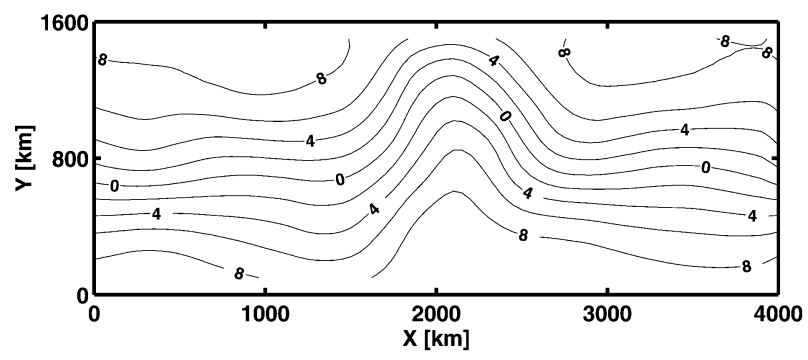

FIG. 8. Estimate of the mean sea surface height after assimilating noisy sea surface height data. Contour interval is $2 \mathrm{~cm}$. Compare to Fig. 2.

\section{Gyre experiments with noisy sea surface height data}

To demonstrate the behavior of the system in a regime in which the the topography has a smaller impact on the flow than in a zonal channel, attention is now turned to a closed basin with a gyre flow. Dimensions are 4000 $\mathrm{km}$ in both the zonal and meridional direction: the horizontal grid cell length is $200 \mathrm{~km}$, the maximum depth $H_{0}=4 \mathrm{~km}, f_{0} \approx 7.3 \times 10^{-5} \mathrm{~s}^{-1}$, and $\beta \approx 2.0 \times 10^{-11}$ $\mathrm{s}^{-1} \mathrm{~m}^{-1}$. The flow is forced by a stationary zonal wind stress $\tau^{x}=\tau_{0} \cos \pi y / Y$, with $\tau_{0}=10^{-4} \mathrm{~m}^{2} \mathrm{~s}^{-2}$. A linear bottom friction parameter $r=0.04 \mathrm{~m} \mathrm{~s}^{-1}$ leads to a western boundary current that the coarse grid can resolve. An analytical problem analogous to that posed above in Eq. (9) can be formulated. Because of the choice of parameters, the rms amplitude of the sea surface height itself is only of the order of $1 \mathrm{~mm}$ in contrast to $6 \mathrm{~cm}$ in section 5 .

Proceeding numerically again, however, the gyre flow is by far less sensitive to changes in the bottom topography than is the flow in the zonal channel. A meridional midocean Gaussian ridge of 2000-m height and 200-km half-width at the center of the basin modifies the flow primarily away from the western boundary current. With

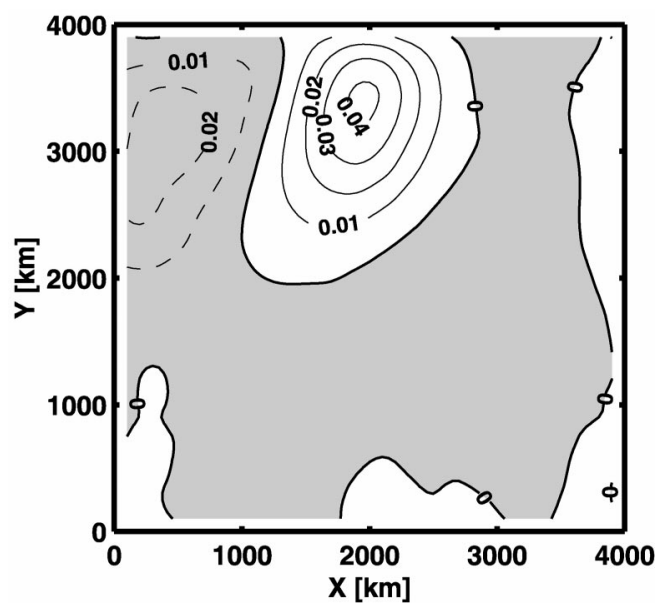

FIG. 10. Initial gradient of objective function (12) with respect to depth for the gyre experiment. Contour interval is $0.01 \mathrm{~m}^{-1}$ (the objective function itself has no units).

the ridge, the zonal steady-state flow along the northern and the southern boundary is topographically steered equatorward (Fig. 9), but it does not follow the geostrophic contours as closely as does the flow in the zonal channel. As a consequence, decreasing the ridge height to $1000 \mathrm{~m}$ above the seafloor leads only to a slight change in the flow. The rms difference between the sea surface height with the full ridge and with the smaller ridge is $0.25 \mathrm{~mm}$, that is, over one order of magnitude smaller than in the experiments of section 5 although the change in topography is considerably larger here.

Treating the true sea surface height as data, the initial gradient of objective function (12) with respect to depth for the model with the smaller ridge is plotted in Fig. 10. The prior rms error estimate for sea surface height is $1 \mathrm{~mm}$. The objective function is most sensitive to depth over the northern part of the ridge where increas-
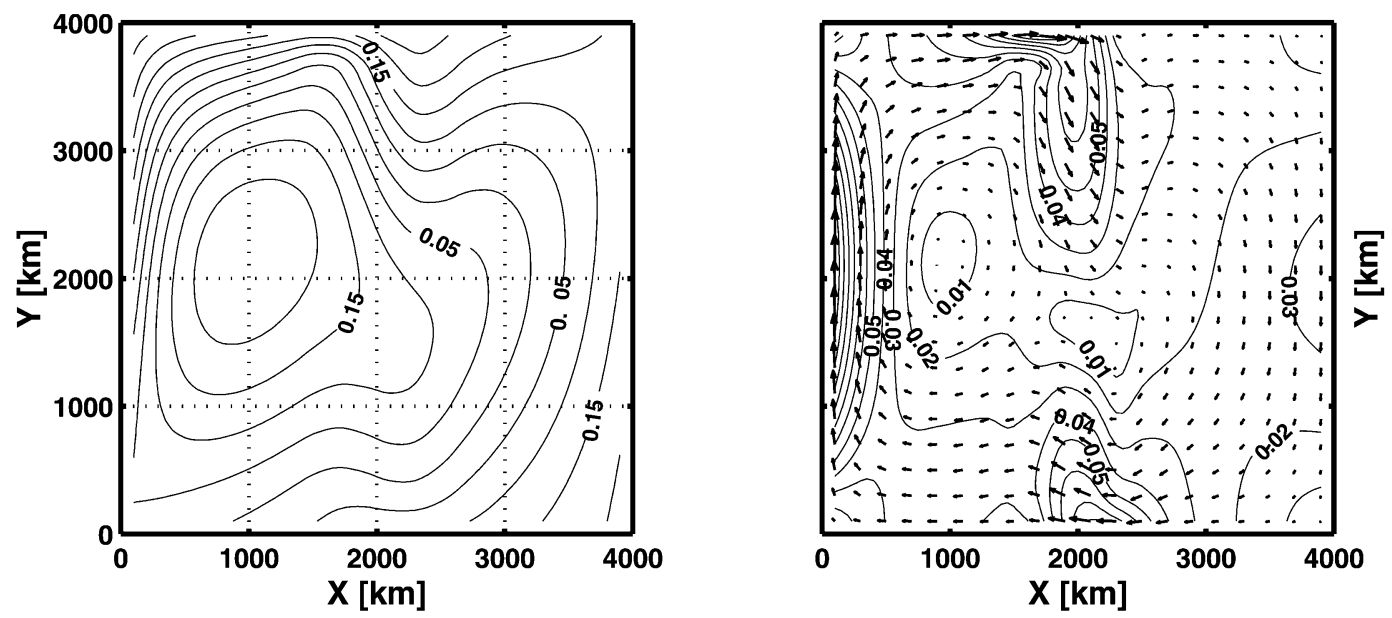

FIG. 9. (left) Sea surface height (in $\mathrm{cm}$ ) and (right) velocity field (contours in $\mathrm{cm} \mathrm{s}^{-1}$, arrows indicate direction of flow) of the gyre with the full ridge of $2000 \mathrm{~m}$ along $x=2000 \mathrm{~km}$. 

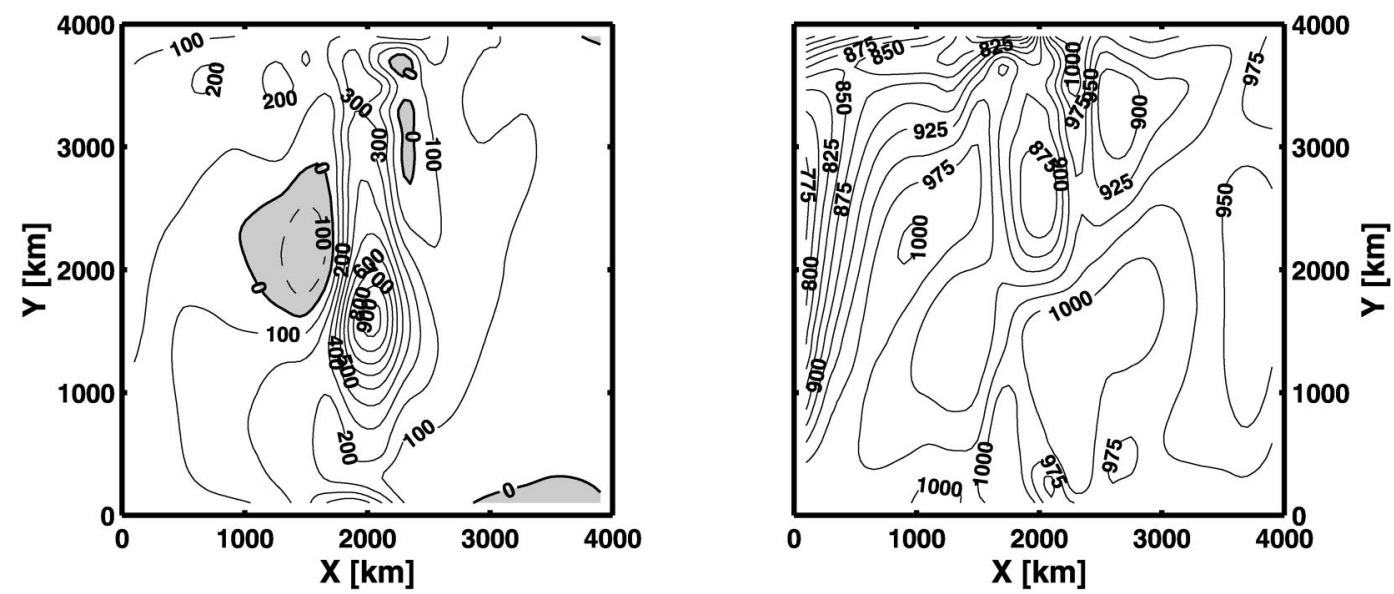

FIG. 11. (left) True errors (difference between estimated and true depth, contour interval is $100 \mathrm{~m}$ ) and (right) estimated errors (square root of the diagonal of the inverse Hessian, contour interval is $25 \mathrm{~m}$ ) of topography in meters, for the case of error-free sea surface height data. The height of the ridge is initially $1000 \mathrm{~m}$. Deviations from the (false) initial estimate are penalized.

ing the ridge height would deflect the flow further equatorward and thus decrease the model-data misfit.

An eigenvalue decomposition of the Hessian of the objective function, similar to that of section 4 , reveals that - apart from the grid-scale components-the eigenvectors corresponding to the smallest eigenvalues (the least constrained shapes) have a peak near the point ( $x$, $y) \approx(1000 \mathrm{~km}, 2000 \mathrm{~km})$ where there is little flow.

\section{a. Error-free sea surface height data}

The forward model is started with an initial estimate for the ridge height of $1000 \mathrm{~m}$ (extending to 3000-m water depth) when the "correct" value is a height of $2000 \mathrm{~m}$. The model then must recover the true depth from sea surface height "data" that have been generated by the model with the "correct" ridge height of 2000 $\mathrm{m}$. The deviations from the (false) initial estimate are penalized as described by objective function $g_{2}$ [Eq. (13)]. The prior errors in this experiment are $\sigma_{\eta}=1$ $\mathrm{mm}$ and $\sigma_{h}=1000 \mathrm{~m}$.

The solution after 48 minimization steps is depicted in Fig. 11. As before, the solution resolution is highest where the current velocities are high. The true errors (difference between estimated and true depth on the lefthand side of Fig. 11) are greatly reduced over the northern and southern end of the ridge whereas at the center of the gyre, where the fluid is almost at rest, the ridge is poorly recovered. Estimated errors, which are consistently larger than the true errors, show their minimum in the western boundary current. A local error minimum is also observed in the north where the ridge causes the strongest deflection of the flow. The overall error reduction is small compared to the uniform prior error of $1000 \mathrm{~m}$. A larger estimated uncertainty remains and it is concluded that sea surface height data in this configuration can only weakly constrain the topography.

\section{b. Noisy sea surface height data}

Gaussian noise with a standard deviation of $1 \mathrm{~mm}$ is added to the sea surface height data and the experiment of section 6a is repeated. Convergence is slow, so that the norm of the gradient is only reduced by a factor of 0.04 after 300 minimization steps. The rms difference between the estimated and the true depth is actually increased from $298 \mathrm{~m}$ before the optimization to $439 \mathrm{~m}$ afterward (not shown). The model cannot find a depth estimate that is consistent with prior assumptions or the true solution. Apparently the initial estimate of topography corresponds to a point in the phase space that is too far away from the global minimum, so that the nonlinear minimization with noisy data slowly converges to a local minimum. Further experiments, in which the initial depth estimate has been moved closer to the true depth, confirm this conclusion: for an initial height estimate of the ridge of $1500 \mathrm{~m}$, the optimization converges quickly and the solution is consistent with the true depth within estimated errors (Fig. 12).

To overcome the poor convergence of the optimization problem with an initial ridge height of $1000 \mathrm{~m}$, a smoothness requirement, a simple way of demanding a spatially correlated solution, is added to the objective function. Correlation is achieved by adding nondiagonal contributions to the depth weight matrix in the objective function $\mathcal{I}_{2}$, here constructed from the matrix operator $\mathbf{L}$ that represents the discretized Laplacian operator,

$$
\mathbf{C}_{h}^{-1}=\sigma_{h}^{-2} \mathbf{I}+\sigma_{r}^{-2} \mathbf{L}^{\mathrm{T}} \mathbf{L} .
$$

With the new objective function $g_{2}$, in which $\sigma_{r}=$ $2 \sigma_{h} \Delta x^{-2}$ is chosen such that both terms in Eq. (14) have the same order of magnitude, faster convergence is achieved: the norm of the gradient is reduced by a factor of $10^{5}$ after 59 minimization steps. Also the rms difference between the estimated and the true depth is de- 

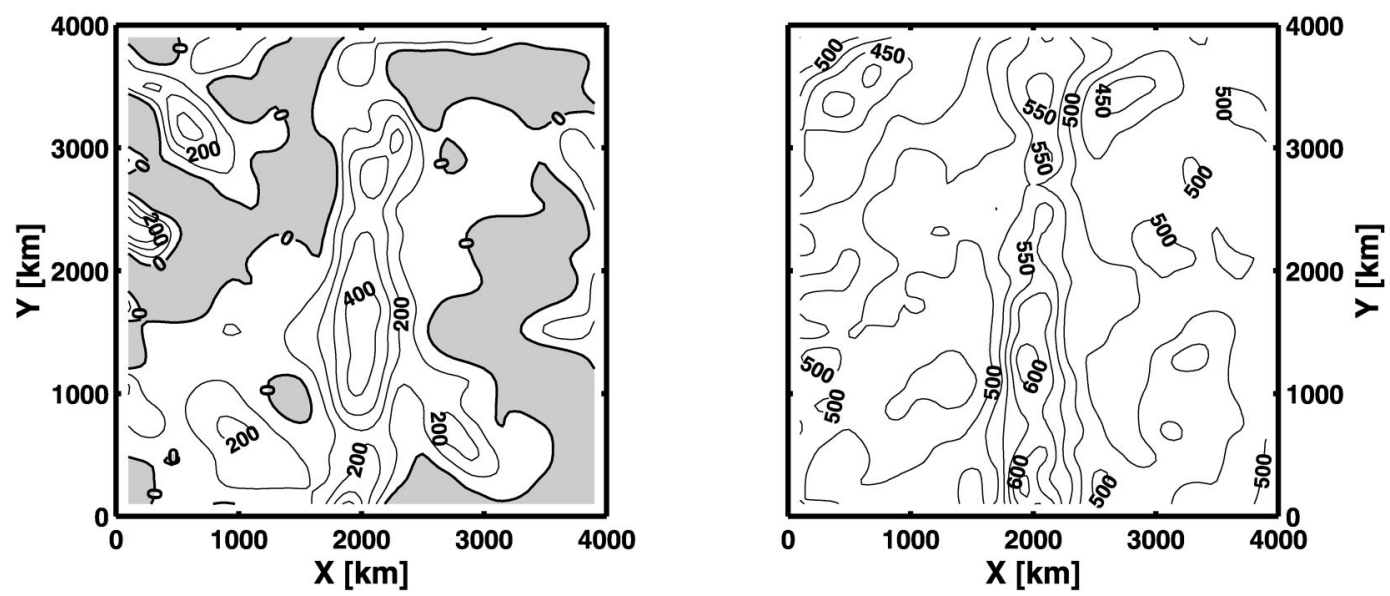

FIG. 12. (left) True errors (difference between estimated and true depth, contour interval is $100 \mathrm{~m}$ ) and (right) estimated errors (square root of the diagonal of the inverse Hessian, contour interval is $25 \mathrm{~m}$ ) of topography in meters, for the case of noisy sea surface height data. The height of the ridge is initially $1500 \mathrm{~m}$. Deviations from the (false) initial guess are penalized. To obtain convergence in this solution, the initial guess has been moved closer to the true depth than for the solutions plotted in Figs. 11 and 13.

creased to $275 \mathrm{~m}$, but the spatial patterns of both the true and estimated errors show less resemblance with the flow field and the topographic features as in the perfect-data case (Fig. 13). More than $90 \%$ of the true errors are smaller than one standard deviation of the estimated errors, and all true errors are smaller than two estimated standard deviations.

\section{Discussion and conclusions}

In a steady barotropic situation, it is practical to treat the bottom topography as a control variable to be determined from the combination of a model and observations. This conclusion is consistent, despite the radically different physical setting, with shallow water gravity wave studies (e.g., Das and Lardner 1992; ten
Brummelhuis et al. 1993). Here, we put this concept into the context of modeling the general circulation of the ocean.

Surface elevation alone does not, empirically, produce a full uniquely determinable estimate of the bottom topography of the underlying model, especially when the data are noisy and small eigenvalues become part of the effective null space. As with all such problems, the accuracy of the a priori information has a direct influence on the final accuracy and precision of the results. The only simple generalization is that regions of high flow velocity tend to produce better results, with weak flow regions providing relatively little information about the topography. A Southern Ocean-like channel shows a greater sensitivity of the elevation to topographic perturbations, and this sensitivity translates into
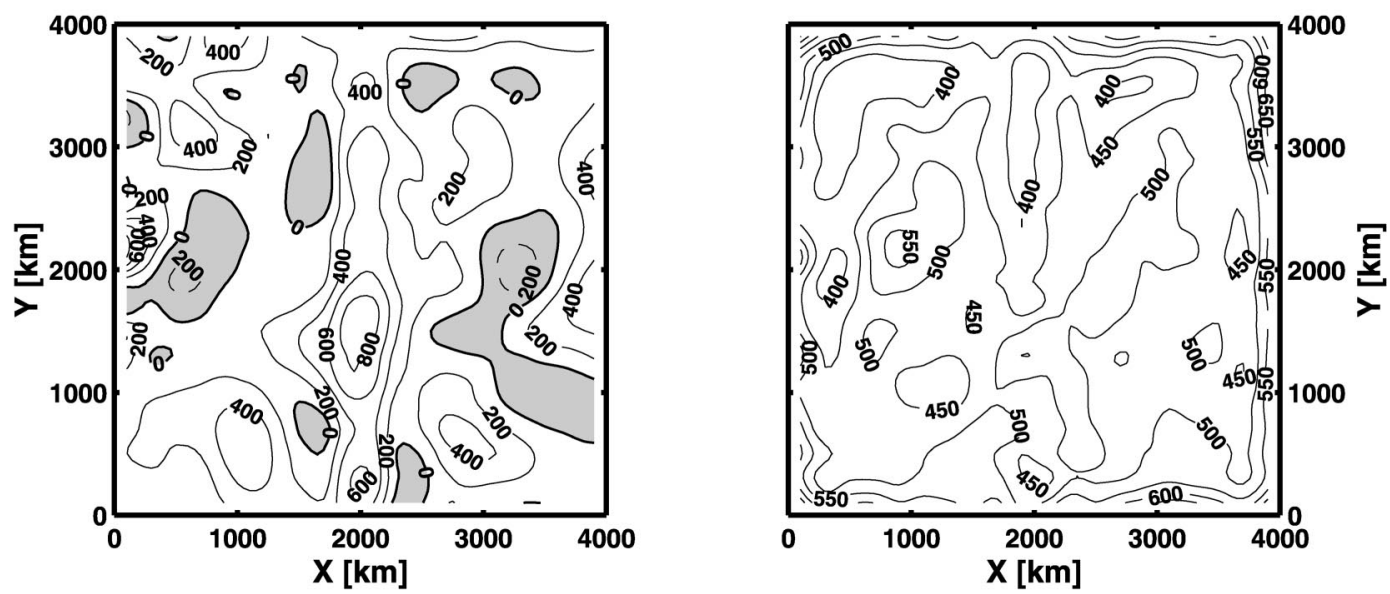

FIG. 13. (left) True errors (difference between estimated and true depth, contour interval is $200 \mathrm{~m}$ ) and (right) estimated errors (square root of the diagonal of the inverse Hessian, contour interval is $50 \mathrm{~m}$ ) of topography in meters, for the case of noisy sea surface height data with penalized roughness. The height of the ridge is initially $1000 \mathrm{~m}$. Deviations from the (false) initial estimate are penalized as well. 
a greater ability to recover the topography. In midlatitude gyre problems, errors in the bottom topography are unlikely to be the dominant source of model problems, given all of the other uncertainties involved. To that extent, the insensitivity there is good news.

The above conclusion seems also valid if the accuracy of the, in our case, sea surface height data is dramatically increased with the new space-borne gravity missions, for example, the Gravity Recovery and Climate Experiment mission (GRACE) (Tapley 1997). In our model we can reduce the formal error to zero as in section 4 , but there still remains a null space, which is associated with regions of weak flow.

When attention is turned to more realistic problems, particularly those involving baroclinicity, a number of major changes can be anticipated. The sensitivity of the surface elevation to the depth structure in general will decrease in a model that allows for baroclinic compensation (e.g., Holland 1973; Marshall and Stephens 2001). On the other hand, topography can have a major impact on water mass properties, which are a central oceanographic observable and for which there exists a major database. Undoubtedly there will be technical details of deducing the best-fitting topography in such models, but the principle of doing so does not seem to be in doubt. A likely major issue is the expected coupling between topographic representation and mixing parameterizations.

Also unexplored at this time is the information content, in the barotropic model, of the time-dependent motions that are readily observed with altimeters. This step is left to the future as well.

Technical points raised by the use of topography as a control variable have been glossed over here, on the basis that numerically, no particular difficulties were encountered. On a more theoretical level, particularly if one returns to the analytical form of Eq. (7), the existence of the adjoint might be called into question. In the continuous formulation, the existence of the adjoint model depends upon the differentiability of the forward model with respect to the topographic field. In practice, as here, with a little care, discrete models do not produce numerical derivatives that are infinite. Experience with the adjoint tool used does show that it is possible to write FORTRAN codes that cannot be compiled, but these problems usually result from coding issues, rather than from mathematical ones.

Here, as a particular example, we explicitly avoided the use of code that fails to be numerically differentiable. For a finite volume discretization of the equations of motion as in the MITgcm (Marshall et al. 1997), where the depth at the interface between two grid cells (velocity points on a $\mathrm{C}$ grid) is evaluated as the minimum of the two cell depths (tracer points), the FORTRAN minimum function is not differentiable when the grid cells have exactly the same depth. In this situation-which is common in ocean general circulation models where the abyssal plains are characterized by many grid cells with exactly the same depth-the discontinuities of the gradients of the objective function may cause numerical difficulties in the minimization. One remedy may be the shaved cell formulation, where the step topography of the partial cells with flat bottoms is replaced by piecewise linear topography (Adcroft et al. 1997).

The use of bottom topography as a control variable is only the first of a number of unorthodox controls that need to be explored. Prominent among these are the inference of lateral and bottom boundary conditions from observations. Open boundaries are already being employed in state estimation, where inflows, outflows, and scalar properties being carried become parts of the control vector (e.g., Zhang and Marotzke 1998). But even with models using conventional Laplacian friction, one has to make a choice between free-slip and no-slip (Adcroft and Marshall 1998). In models with, for example, biharmonic terms in the momentum equation, the most appropriate higher-order boundary conditions are little more than guesses. The distinction between open and closed boundaries is thus seen to be somewhat artificial. Eventually, bottom topography as well as all boundary conditions, are expected to be included generally as a control variable in general circulation models of arbitrary complexity. Much remains to be done.

Acknowledgments. ML would like to thank Samar Khatiwala and Patrick Heimbach for many fruitful discussions. The authors were supported in part by the ECCO Consortium of the National Oceanographic Partnership Program (ONR, NSF, NASA).

\section{REFERENCES}

Adcroft, A., and D. Marshall, 1998: How slippery are piecewiseconstant coastlines in numerical ocean models? Tellus, 50A, 95108.

C. Hill, and J. Marshall, 1997: Representation of topography by shaved cells in a height coordinate ocean model. Mon. Wea. Rev., 125, 2293-2315.

Das, S. K., and R. W. Lardner, 1991: On the estimation of parameters of hydraulic models by assimilation of periodic tidal data. $J$. Geophys. Res., 96 (C8), 15 187-15 196.

_ dimensional numerical tidal model. Int. J. Numer. Methods Fluids, 15, 313-327.

Ganachaud, A., C. Wunsch, M.-C. Kim, and B. Tapley, 1997: Combination of TOPEX/Poseidon data with a hydrographic inversion for determination of the oceanic general circulation and its relation to geoid accuracy. Geophys. J. Int., 128, 708-722.

Giering, R., and T. Kaminski, 1998: Recipes for adjoint code construction. ACM Trans. Math. Software, 24, 437-474.

Gilbert, J. C., and C. Lemaréchal, 1989: Some numerical experiments with variable-storage quasi-Newton algorithms. Math. Program., 45, 407-435.

Heemink, A. W., E. E. A. Mouthaan, M. R. T. Roest, E. A. H. Vollebregt, K. B. Robaczewska, and M. Verlaan, 2002: Inverse 3D shallow water flow modelling of the continental shelf. Contin., Shelf Res., 22, 465-484.

Holland, W. R., 1973: Baroclinic and topographic influences on the transport in western boundary currents. Geophys. Fluid Dyn., 4, $187-210$. 
Il'in, A. M., V. M. Kamenkovich, V. F. Kanayev, T. G. Zhugrina, and L. I. Lavrishcheva, 1974: An experiment in constructing a smoothed bottom relief of the World Ocean (in Russian). Okeanologiya, 14, 617-622.

Lardner, R. W., A. H. Al-Rabeh, and N. Gunay, 1993: Optimal estimation of parameters for a two-dimensional hydrodynamical model of the Arabian Gulf. J. Geophys. Res., 98 (C10), 18 22918242.

LeGrand, P., 2001: Impact of the Gravity Field and Steady-State Ocean Circulation Explorer (GOCE) mission on ocean circulation estimates: Volume fluxes in the climatological inverse model of the Atlantic. J. Geophys. Res., 106 (C9), 19 597-19610.

Marotzke, J., R. Giering, K. Q. Zang, D. Stammer, C. Hill, and T. Lee, 1999: Construction of the adjoint MIT ocean general circulation model and application to Atlantic heat transport sensitivity. J. Geophys. Res., 104 (C12), 29 529-29 547.

Marshall, D. P., and J. C. Stephens, 2001: On the insensitivity of the wind-driven circulation to bottom topography. J. Mar. Res., 59, $1-27$.

Marshall, J., A. Adcroft, C. Hill, L. Perelman, and C. Heisey, 1997: A finite-volume, incompressible Navier Stokes model for studies of the ocean on parallel computers. J. Geophys. Res., 102 (C3), 5753-5766.

McFarlane, N. A., 1987: The effect of orographically excited gravity wave drag on the general circulation of the lower stratosphere and troposphere. J. Atmos. Sci., 44, 1775-1800.

NOAA, 1988: Digital relief of the surface of the Earth. Data Announcement 88-MGG-02, NOAA/National Geophysical Data Center, Boulder, CO.
Pedlosky, J., 1987: Geophysical Fluid Dynamics. Springer, 710 pp. Schröter, J., M. Losch, and B. M. Sloyan, 2002: Impact of the Gravity Field and Steady-State Ocean Circulation Explorer (GOCE) mission on ocean circulation estimates 2. Volume and heat fluxes across hydrographic sections of unequally spaced stations. $J$. Geophys. Res., 107, 3012, doi:10.1029/2000JC000647.

Smith, W. H. F., and D. T. Sandwell, 1997: Global seafloor topography from satellite altimetry and ship depth soundings. Science, 277, 1957-1962.

Stammer, D., and Coauthors, 2002: The global ocean circulation during 1992-1997, estimated from ocean observations and a general circulation model. J. Geophys. Res., 107, 3118, doi:10.1029/ 2001JC000888.

Tapley, B. D., 1997: The Gravity Recovery and Climate Experiment (GRACE). EOS, Trans. Amer. Geophys. Union, 78 (Suppl.), 163.

ten Brummelhuis, P. G. J., A. W. Heemink, and H. F. P. van den Boogaard, 1993: Identification of shallow sea models. Int. J. Numer. Methods Fluids, 17, 637-665.

Thacker, W. C., 1989: On the role of the Hessian matrix in fitting models to data. J. Geophys. Res., 94 (C5), 6177-6196.

Wunsch, C., 1996: The Ocean Circulation Inverse Problem. Cambridge University Press, 442 pp.

__ , and D. Stammer, 1998: Satellite altimetry, the marine geoid, and the oceanic general circulation. Ann. Rev. Earth Planet. Sci., 26, 219-253.

Zhang, K. Q., and J. Marotzke, 1998: The importance of open-boundary estimation for an Indian Ocean GCM-data synthesis. J. Mar. Res., 57, 305-334. 